\title{
The Challenge of Mobile Devices for Human Computer Interaction
}

\author{
MARK DUNLOP ${ }^{1}$, STEPHEN BREWSTER $^{2}$ \\ ${ }^{1}$ Department of Computing and \\ Information Systems, University of \\ Strathclyde, Glasgow, G1 1XQ, UK \\ mark.dunlop@cis.strath.ac.uk \\ Phone: +44 (0)141 5483497 \\ Fax: +44(0)1415525330
}

\author{
${ }^{2}$ Department of Computing Science, \\ University of Glasgow, Glasgow, \\ G12 $8 Q Q, U K$ \\ stephen@dcs.gla.ac.uk \\ Phone +44(0)141330 4966 \\ Fax +44 $141(0) 3304913$
}

www.cis.strath.ac.uk/ mdd/mobilehci01/

Current mobile computing devices such as palmtop computers, personal digital assistants (PDAs) and mobile phones, and future devices such as Bluetooth and GSM enabled cameras, and music players have many implications for the design of the user interface. These devices share a common problem: attempting to give users access to powerful computing services and resources through small interfaces, which typically have tiny visual displays, poor audio interaction facilities and limited input techniques. They also introduce new challenges such as designing for intermittent and expensive network access, and design for position awareness and context sensitivity.

No longer can designers base computing designs around the traditional model of a single user working with a personal computer at his/her workplace. In addition to mobility and size requirements, mobile devices will also typically be used by a larger population spread than traditional PCs and without any training or support networks, whether formal or informal. Furthermore, unlike early computers which had many users per computer, and PCs with usually one computer per user, a single user is likely to own many mobiles devices [1] which they interact with indifferent ways and for different tasks.

Mobile devices present human-computer interaction (HCI) designers with five main challenges:

1. Designing for mobility: As users are mobile they will not have many of the props around them to support work (e.g. notes on desks), will need to work with small devices, are likely to have a far from ideal working environment and this environment will change drastically as the user moves;

2. Designing for a widespread population: Users will not normally have any formal training in their technologies and consider them as devices to be used rather than computers to be maintained;

3. Designing for limited input/output facilities. Screen sizes will improve in resolution in terms of colour support and pixels per $\mathrm{cm}$, but will always be small 
due to the need for portability. Sound output quality is often very poor with restricted voice recognition on input. Keyboards are limited in size and number of keys and other pointing devices are often hard to use when on the move.

4. Designing for (incomplete and varying) context information: Through various sensors and networks, mobile devices can be made aware of their context (e.g. current location through the Global Positioning System). This gives new information to the systems but brings problems of implying task and user level activities from sensor information and unreliable or patchy sensor coverage. Work on position aware tourism guides, for example, highlight many of these problems.

5. Designing for users multitasking at levels unfamiliar to most desktop users: Multitasking and support for task interruption is one of the keys to successful desktop design, with mobile devices the opportunities for and frequency of interruptions are likely to be much higher given the environments in which the devices will be used.

This special issue of Personal and Ubiquitous Computing is based on the outcomes of the Third International Workshop on Human Computer Interaction with Mobile Devices (Mobile HCI '01 held in Lille on $10^{\text {th }}$ September 2001 in conjunction with IHM-HCI 2001 [2]). The Mobile HCI workshop series ${ }^{1}$ provides a forum for academics and practitioners to discuss the challenges and potential solutions for effective interaction with mobile systems. It is intended to cover the design, evaluation and applications of techniques and approaches for all mobile computing devices. Previous workshops were held in Glasgow in 1998 and at INTERACT 99 in Edinburgh. The next workshop will take place in Pisa in 2002.

During the workshop a range of papers, posters and demonstrations were presented. The authors of the papers were invited to resubmit them for inclusion in this special issue; twelve were submitted and ten were chosen after re-review.

The range to topics covered by this special issue is broad. Several papers considered interaction techniques for mobiles. Chittaro and Cin look at different menu item selection techniques in WAP interfaces, De Bruijn, Spence and Chong use the RSVP technique to improve Web browsing on small devices, and Holland, Morse and Gedenryd describe a completely auditory interface for a GPS receiver. Rist and Brandmeier attempt to solve the problem of presenting usable images on small, limited display devices, such as WAP phones, by testing a range of image transformations.

Three papers presented underlying software technologies to support design and use of mobile devices. Context detection for mobile users plays a major role in enabling novel, human-centric interfaces. Michahelles and Samulowitz give an architecture to support context sensitivity in a sensor network. Cherverst, Mitchell and Davies describe a tourist information system and how they tried to design using context information. They 
discuss the problems of trying to meet the conflicting goals of adapting to changes in context while at the same time making the interface predictable. Roth presents the use of software patterns to help in the efficient design of mobile applications and systems.

Izadi et al. describe the Citywide project that is exploring ways in which technology can provide people with rich and engaging digital experiences as they move through physical space, including historical experiences, performances and games. Iacucci and Kuutti present a novel technique for capturing requirements of mobile systems using theatrical and role-playing techniques and Strom presents a different viewpoint on personal mobile devices and the user's relationship with them.

\section{Acknowledgements}

Thanks to all the authors who submitted excellent papers and reviewers who put time and energy into their reviews. Thanks also to the participants for making it such an interesting and enjoyable day. In particular, thanks to David McGookin from Glasgow who worked hard doing the registrations and organisation behind the scenes. The workshop was sponsored by the British HCI group, GIST at Glasgow and EPSRC project GR/L66373.

\section{References}

1. D. A. Norman, The Invisible Computer, MIT Press, 1999.

2. Blandford, A., Vanderdonckt, J. and Gray, P., Eds. People and Computers XV - Interaction without frontiers. Springer, London, 2001. 